\title{
Weighted transitivity scores that account for triadic edge similarity in undirected graphs
}

Running title: Weighted graph transitivity scores for animal contact networks

\begin{abstract}
The graph transitivity measures the probability that adjacent vertices in a network are interconnected, thus revealing the existence of tightly connected neighborhoods playing a role in information and pathogen circulation. When the connections vary in strength, focusing on whether connections exist or not can be reductive. I score the weighted transitivity according to the similarity between the weights of the three possible links in each triad. I illustrate the biological relevance of that information with two reanalyses of animal contact networks. In the rhesus macaque Macaca mulatta, a species in which kin relationships strongly predict social relationships, the new metrics revealed striking similarities in the configuration of grooming networks in captive and free-ranging groups, but only as long as the matrilines were preserved. In the barnacle goose Branta leucopsis, in an experiment designed to test the long-term effect of the goslings' social environment, the new metrics uncovered an excess of weak triplets closed by strong links in males compared to females, and consistent with the triadic process underlying goose dominance relationships.
\end{abstract}

Key-words: animal social network; indirect interactions; clustering coefficient; valence; graph theory; social style; intransitivity; graph permutation; socio-spatial structure 


\section{Introduction}

2 Social interactions between individuals are rarely binary. Instead, the connections between

3 individuals vary in strength or valence. And even if the instant interactions indeed have a binary

4 outcome, the consequences usually accumulate over several interactions, e.g., the fitness benefits of

5 long-lasting bonds (Silk et al., 2009), and the hierarchy emerging from successive agonistic

6 interactions (Franz et al., 2015). In that context, it seems useful to develop network summary metrics

7 that account for edge weights (Barrat et al., 2004), instead of dichotomizing the network or to scale

8 the timeframe down until interactions can be considered binary. Here I focus on the graph

9 transitivity (Newman et al., 2002). The graph transitivity answers the question: when A is separately

10 connected to $B$ and to $C$, what is the probability that $B$ and $C$ are also connected. The transitivity

11 influences the occurrence of indirect interactions (when B and C are not connected) as well as the

12 expected shortest path length influencing the circulation of pathogens and information.

13 In its usual definition for binary networks, the graph transitivity corresponds to the relative

14 frequency of closed and open triplets (Newman, Watts, \& Strogatz, 2002; see also Watts \& Strogatz

15 (1998) and Barrat et al. (2004) for different definitions that use vertex-level scores instead of triplet

16 enumeration).

\begin{tabular}{|l|l}
\hline Eq. 1 & $\mathcal{C}_{0}=\frac{\mathcal{T}_{\text {closed }}}{\mathcal{T}}=\frac{1}{\mathcal{T}} \sum \omega^{(0)}=\frac{\operatorname{tr}\left(\boldsymbol{B}^{3}\right)}{\left.\sum_{i \neq j} \boldsymbol{B}^{2}\right|_{i, j}}$
\end{tabular}

The sum in the middle is over triplets, i.e., any three individuals with at least two non-null links

between them. $\mathcal{T}$ is the total number of triplets in the network, $\mathcal{T}_{\text {closed }}$ is the number of closed

triplets among these. $\omega^{(0)}$ equals 1 if the triplet is closed and 0 if the triplet is open. Note that each

closed triangle $(a, b, c)$ contributes three closed triplets $(a, b),(b, c)$, and $(a, c)$ (Fig. 1). The last term 
In weighted networks, the distribution of edge weights can significantly modify the transitive properties. For example, a triangle that features two strong links and one weak link may functionally operate more like an open triplet. Inversely, weak links that would maybe be considered insignificant when dichotomizing the network for transitivity evaluation may actually perform a significant role.

There already exists a weighted version of $\mathcal{C}_{0}$, derived by Opsahl and Panzarasa (2009). They use the geometric mean of the two links in each triplet as the weight of that triplet: $\omega^{(1)}(a, b)=\sqrt{a b}$ (or alternatively, the arithmetic mean, or the minimum, or the maximum). Then they derive the weighed transitivity as follows.

$$
\begin{array}{|l|l}
\hline \text { Eq. } 2 & \mathcal{C}_{1}=\frac{\sum_{\text {closed }} \omega^{(1)}}{\sum_{\text {open }} \omega^{(1)}+\sum_{\text {closed }} \omega^{(1)}}=\frac{\operatorname{tr}\left(f\left(\boldsymbol{A}^{2} \boldsymbol{B}\right)\right)}{\left.\sum_{i \neq j} f\left(\boldsymbol{A}^{2}\right)\right|_{i, j}}
\end{array}
$$

The second term represents a matrix formulation where $\boldsymbol{A}$ is the weighted adjacency matrix featuring

the strength of the link between $i$ and $j$ in cell $(i, j), B$ is the dichotomized version of $\boldsymbol{A}$, and $f$ is a

function that takes the square root of each cell.

Eq. 2 is attractive because $\mathcal{C}_{1}$ verifies most of the properties of $\mathcal{C}_{0}$ (Opsahl and Panzarasa, 2009). The main use of $\mathcal{C}_{1}$ is to assess the effect of the triplet weight on the probability that the third link in the triplet is created (Opsahl and Panzarasa, 2009). If $\mathcal{C}_{1}>\mathcal{C}_{0}$, strong triplets (with a high $\omega^{(1)}$ ) are more frequently closed than weak triplets (with a low $\omega^{(1)}$ ). Inversely, if $\mathcal{C}_{1}<\mathcal{C}_{0}$, then weak triplets are more frequently closed than strong triplets.

My grievances with Eq 2 are: (i) Eq. 2 does not capture the information about the similarity between the links: $a$ and $b$ can be very different or very similar, and yet yield the same $\omega^{(1)}$ value. (ii) Eq. 2 still involves some dichotomization: the strength of the closing link does not influence the triplet weight (Barrat et al., 2004). The only way to assess the effect of the weight of the third link, in an ad hoc way, would be to change the threshold $c^{*}$ that is used to dichotomize the closing link. In addition to being relatively cumbersome and hard to interpret biologically, this procedure is expectedly unstable owing to the decrease in sample size when $c^{*}$ increases. It also introduces an artificial nonlinearity at 
the threshold. Moreover, there rarely exists enough information to decide on a biologically meaningful threshold. The choice of $c^{*}$ is often arbitrary (e.g., "more than one contact", "statistically significant association”).

I propose new scores designed to capture the information about the similarity between the three edge weights in each triad, without any dichotomization required. I replaced the triplet weights $\omega^{(0)}$ and $\omega^{(1)}$ by a measure of the imbalance between the three links $a, b$, and $c$. I identified two ways to do so. The first is the rescaled sum of proportional pairwise differences:
Eq. 3
$\omega=\frac{a-c}{a}+\frac{b-c}{a}-1=\frac{b-2 c}{a}$

The rationale is that $\omega$ compares $c$ to $a$ and to $b . \omega=0$ for a false triplet $(b=c=0), \omega=1$ for an open triplet made of two equally strong links, and $\omega=-1$ for a closed triangle made of three equally strong links (Fig. 1). The second option is the Gini coefficient:

$$
\begin{array}{l|l}
\text { Eq. } 4 \\
\qquad G=\frac{1}{2} \frac{(a-b)+(b-c)+(a-c)}{a+b+c}=\frac{a-c}{a+b+c}
\end{array}
$$

The rationale is that the Gini coefficient is a classical measure of inequality, expected to capture the three-way relationship between $a, b$ and $c . G=1$ for a false triplet $(b=c=0), G=0.5$ for an open triplet made of two equally strong links, and $G=0$ for a closed triangle made of three equally strong links (Fig. 1). Note that throughout, the strengths of the links $a, b$, and $c$ are rescaled to vary between 0 (no link) and 1 (strongest recorded link).

There is covariation between $\omega$ and $G$, and in particular, owing to the constraint that $1 \geq a \geq b \geq$ $c \geq 0$, the domain of possible combinations $(\omega, G)$ is restricted (Fig. 1). Despite this covariation, the two metrics are complementary and need to be interpreted together. For example, $\omega$ tends towards zero either when the triad is a false triplet dominated by a single link, or when the triad is a closed triangle but one of the links is about half as strong as the other two (Fig. 1). The value of $G$ separates between the two situations (Fig. 1). 
67

In other words, the aim of the method is to reduce the 3D space $\{a, b, c \mid 1 \geq a \geq b \geq c \geq 0\}$ into a 2D space $\{\omega, G \mid 1 \geq a \geq b \geq c \geq 0\}$, for inference about triadic association patterns.

For interpretation purposes, I suggest to divide the $\{\omega, G\}$ space into four quadrants (Fig. 1). I devised a permutation procedure, hereafter termed the quadrant test (Appendix S1), to assess whether the observed distribution of triads in the $\{\omega, G\}$ space depends only on the dyadic edge weights, or also depends on triadic association patterns. When the quadrant test is negative, it means that most of the observed variation in $(\omega, G)$ comes from the dyadic edge weights. When it is positive, triadic associations are biased towards specific combinations of edge weights.

Importantly, this permutation procedure removes all triadic processes. This would include automatic associations based on the finitude of shared resources. More precisely, when the edge weights are computed based on the proportion of time spent in close proximity, or on the proportion of a budget spent on joint ventures, transitivity may arise mechanistically. If individual A spends most of its time with $B$ and $C$, then mechanistically $B$ and $C$ also spend most of their time together, even if they were here for A not for each other (Granovetter, 1973). Therefore, the interpretation of the quadrant test as formulated above critically depends on the way the edge weights are computed and also on the realized, empirical distribution of dyadic edge weights.

Next, one can compute graph-level transitivity scores based on $\omega$ and $G$. They are simply the average of $\omega$ and $G$ over all the triads.

\begin{tabular}{|l|l}
\hline Eq. 5 & $\mathcal{C}_{\omega}=\frac{1}{\tilde{T}} \sum \omega \quad$ and $\quad \mathcal{C}_{G}=\frac{1}{\tilde{T}} \sum G$ \\
\hline
\end{tabular}

$\tilde{\mathcal{T}}$ is the number of triads (a triad being any three individuals with at least one non-null link between them, counting one triad per triangle). Note that in Eq. 1 and 2 the sum was over triplets (a triplet being any three individuals with at least two non-null links between them, counting three triplets per triangle). The reason for this change is that the missing edges are now considered as an edge with weight zero. Therefore, despite the parallelism of form, $\mathcal{C}_{\omega}$ and $\mathcal{C}_{G}$ do not compare directly to $\mathcal{C}_{0}$ or $\mathcal{C}_{1}$. 
91 To assess the null hypothesis that the observed transitivity scores are expected from the edge

92 density and edge weight distribution, I devised another permutation procedure, hereafter termed

93 the distance test (Appendix S1). This test is similar in philosophy to the comparison between the

94 usual transitivity score $\mathcal{C}_{0}$ and the edge density $\mathcal{D}_{0}$. I used the Erdős-Rényi model to generate a large

95 number of random networks with the same number of vertices, same number of edges, and the

96 same distribution of edge weights as the focal network (routine sample_gnm in igraph; Csardi \&

97 Nepusz, 2006). Next I computed the Mahalanobis distance between the observed $\left(\mathcal{C}_{\omega}, \mathcal{C}_{G}\right)$ value and the distribution of $\left(\mathcal{C}_{\omega}, \mathcal{C}_{G}\right)$ among the simulated graphs. I computed the p-value of that distance under a chi-squared test with 2 degrees of freedom. If the test is positive, the observed transitivity scores are statistically different from the expectation at random. Note that extreme values of $\left(\mathcal{C}_{\omega}, \mathcal{C}_{G}\right)$, in the lower left or lower right corners of the domain of definition, tend to test positive because the randomization is constrained in these corners. In addition, like for the quadrant test, the interpretation of the distance test critically depends on the way the edge weights are computed, and in particular whether the edge weights involve the expenditure of a finite shared resource like time or money (cf. quadrant test above).

In the following, I report on a simulation study and on two case studies to demonstrate the biological relevance of the new scoring method.

\section{Material and methods}

\section{Simulation study}

The envisioned main use of the new method is to diagnose whether a dense graph exhibits an excess of balanced triangles, of weak triplets closed by strong links, or of strong triplets closed by weak links. To illustrate that this task is achieved, I simulated transitive networks with those exact properties. 
114 Scenario 1: excess of balanced triangles. I generated random full networks in which, at random, half

115 of the edges were assigned a weight of 1 and half of the edges were assigned a weight drawn at

116 random between 0 and 0.5 .

117 Scenario 2: excess of strong triplets closed by weak links. First I generated random uniform networks

118 with a $\mathcal{C}_{0}$-score of 0.8 , using the rguman routine in R-package sna (Butts, 2020). Next, in each

119 triangle, I assigned to one of the three links a low weight, drawn at random between 0 and 0.1 ,

120 whereas the other two links in the triangle still had a large weight of one.

121 Scenario 3: excess of weak triplets closed by strong links. I generated random full networks in which,

122 at random, $85 \%$ of the edges were assigned a weight of 0.1 and $15 \%$ of the edges were assigned a

123 weight drawn at random between 0.75 and 1.

124 In addition to the three scenarios above, I considered a range of usual network types just to explore

125 what their scores would be and whether they would be different from Scenarios 1-3: full networks

126 with uniform or Gaussian distribution of edge weights, half-full networks in which I removed half of

127 the edges at random, lattice graphs, modular graphs, star graphs, and scale-free graphs (Fig. 2). All

128 these were generated using igraph for R (Csardi and Nepusz, 2006).

\section{Illustration 1: grooming networks in rhesus macaques}

Rhesus macaques (Macaca mulatta) are a social primate typically found in multimale groups that can

range in the hundreds of individuals. They exhibit an "intolerant social style" characterized by steep

hypothesis is that the kinship structure of the group, i.e., whether the matrilines are preserved or 
2018). One captive group featured locally born adult and immature offspring, therefore similar to the natural kinship structure (Massen and Sterck, 2013). In the last three groups, all from the same study in captivity, the individuals came from various origins and met each other after weaning age (Balasubramaniam et al., 2018). Edge weights corresponded to the frequency of recorded allogrooming interactions for each dyad during standardized observation sessions. I did not distinguish who was the groomer and who was the recipient, i.e., symmetric, undirected edges.

Illustration 2: barnacle geese experiment

Barnacle geese (Branta leucopsis), like all geese, are among the most gregarious of vertebrates, yet exhibit high levels of competition for food and frequent agonistic behavior (Black and Owen, 1989; Stahl et al., 2001). Within geese flocks, kin and mate support is essential to assert dominance (Black and Owen, 1989; Kurvers et al., 2013). I reanalyzed sub-group memberships in the captive population that Kurvers et al. (2013) maintained in an experimental environment. The edge weights corresponded to the frequency of standardized sampling occasions during which two individuals occurred together on the same feeding patch, as opposed to foraging in separate sub-groups.

My working hypothesis is that individuals have limited control over occasional contacts with flock mates in this captive environment. I interpret the weak links as the baseline level of interaction. By contrast, I interpret the strong links as the expression of social tactics. In these geese, social tactics would involve preferential bonds with familiar individuals from the gosling phase (Kurvers et al., 2013), which seem to replace the strong bond that normally occurs between parents and offspring and between siblings. I hypothesized that when two bonded individuals interacted with the same unfamiliar individual, this created a weak triplet closed by a strong link. Kurvers et al. raised the goslings in two separate flocks of the same size before merging the two flocks together; therefore, half of the individuals were familiar and half were unfamiliar. Another goose social tactic is to spatially avoid the most dominant, heaviest individuals (Stahl et al., 2001). That avoidance tactic can 
164

165

166

167

168

169

170

171

172

173

174

175

176

177

178

competitors both repel a subordinate individual. The original study design afforded the opportunity to compare male and female networks. Although it is a bit tentative, that comparison between sexes might help decipher the two aforementioned proximate mechanisms, because males are more aggressive and exhibit steeper dominance gradients than females.

Throughout, to standardize across datasets and enforce that $0 \leq c \leq b \leq a \leq 1$, I logit-transformed all non-zero weights so that the middle point between the lowest and highest non-zero weights was attributed weight 0.5 . Results were qualitatively the same if using a linear transformation.

\section{Results}

\section{Simulation study}

The new method correctly separated the three test scenarios (Fig. 2b: yellow, golden yellow and orange symbols). It not only distinguished the three scenarios from one another, but from other network types as well.

The usual transitivity scores mostly failed at the same task, although their performance depended on the choice of the threshold $c^{*}$. With no threshold $\left(c^{*}=0\right.$, left panel) or with a high threshold $\left(c^{*}=\right.$ 0.5 , right panel), the usual scores did neither separate the test scenarios from one another, nor from other scenarios, including from scenarios that did not feature any intrinsic excess of one type of triplet. With a moderate threshold (center panel), $\mathcal{C}_{0}$ and $\mathcal{C}_{1}$ separated the three test scenarios by giving them boundary scores 0 or 1 . The value of $c^{*}$ to achieve that separation depended on the specifics of the simulation scenarios, here $c^{*}=0.1$.

\section{Illustration 1: grooming networks in rhesus macaque}

The kinship structure explained more variation in graph-level transitivity scores than the environment (Fig. 3: triangular symbols). However, the edge density (Fig. 3: color scale) was already capturing that information. The new triad-level scores revealed the extent to which both kinship and captivity influenced the transitive properties: $\chi^{2}{ }_{3}$ (kin vs. nonkin) $=3793.8 ; \chi^{2}$ (captive vs. free- 
ranging) $=842.8$ (Fig. 4a-c; both $\mathrm{P}<0.001)$. The difference in transitive properties were much stronger between kin and nonkin groups than between captive and free-ranging groups (Fig. 4a and $4 c$ vs. 4 b). The negative quadrant tests (Fig. 4: filled vs. empty histograms) further revealed that the effect of kinship and captivity on the transitive properties operated via the dyadic edge weights, rather than via specific triadic association patterns. Overall, captivity influenced the frequency of weak links and kinship influenced the frequency of strong links.

\section{Illustration 2: barnacle geese experiment}

Since the networks were full, classical metrics failed at uncovering any other information beyond the fact that edge density $=1$ (Fig. 3a: red round symbol). By contrast, the new metrics revealed differences between the male and female groups (Fig. 3b: "M" vs. "F"). Both sexes exhibited an excess of weak triplets closed by strong links, as predicted, but the effect was much stronger in males than females. This is consistent with the fact that males are more aggressive and more dominant than females, and suggests a role for the spatial avoidance of dominants in the formation of weak triplets closed by strong links. Contrary to the macaque networks, the quadrant test was very significant for the geese (Fig. $4 \mathrm{~d}$ : filled vs. empty histograms; $\mathrm{P}<0.001$ ). This means that the high frequency of weak triplets closed by a strong link but also the high frequency of balanced triangles (Fig. 4d) did not emerge solely from the dyadic association frequencies. Indeed they resulted from triadic-level processes. This result is strongly consistent with the hypothesis of a triad-based dominance mechanism in which bonded individuals are dominant over singletons.

\section{Algorithmic considerations}

The algorithm in Appendix S1 corresponds to the very most basic option. Its complexity is $O\left(V^{3}\right)$, where $V$ is the number of vertices in the graph. Even with parallelization, this becomes intractable for $V>100$, especially if using the aforementioned permutation tests. However, I envision some gains in computing time following Schank and Wagner (2005) and references therein. Note however that most animal datasets feature less than 100 individuals. 


\section{Discussion}

214 I expect these weighted network transitivity scores to be most relevant when 1) the networks are

215 dense, meaning that most dyads interact at least once during the study period and 2) the strength of

216 dyadic connections depends on the strength of other connections in the immediate social

217 neighborhood. The barnacle geese case study provided a real-life example of such a situation where

218 the occurrence of a strong dyadic bond suppressed the probability that other strong dyadic bonds

219 would occur in the immediate neighborhood.

220 The new metrics can also prove useful in large comparative analyses because (1) they capture a

221 different type of information than usual graph summary metrics and (2) they do not require nor

222 depend on the dichotomization of the network using an arbitrary threshold. The appropriate

223 threshold depends on the biological situation (cf. simulation results), making standardization across

224 datasets a bit hazardous. In addition, filtering the statistically insignificant links can be

counterproductive for the inference, because the weakest links in a network critically contribute to

major processes, such as the spread of epidemics and information from clique to clique (Granovetter,

1973). With the metrics proposed here, there is no need to filter the weakest links. For example, my

illustration cases suggest that in captive populations, weak connections represented the baseline

level of contact between companions of captivity. The macaque networks were nevertheless still

dominated by false or nearly false triplets, as expected due to the high selectivity of grooming

interactions. But in the geese, the high baseline rate of contact combined with a few strong bonds

made weak triplets closed by strong links the most frequent type of triad (Fig. 4).

step back in terms of complexity. Indeed, this is an approach geared towards the study of time- 
238 particularly relevant, because repeated interactions are required to take effect and/or because the

239 frequency of dyadic contacts is the best summary for functional analysis. Logistically, aggregating

240 data over some span of time also has the advantage of smoothing out any error, missing data, or

241 variation in sampling effort. Such errors are frequent in datasets collected by people from

242 uncooperative animal subjects. They are expected to decrease in frequency with the advent of

243 automated data collection devices, but the latter remain subject to stringent constraints regarding

244 the definition of dyadic contacts, e.g., depending on the detection distance of RFID transponders. In

245 that context, I envision the next step for the study of aggregated contact networks might be to

246 incorporate them within a time-specific capture-recapture framework (Lebreton et al., 2009). This

247 means monitoring the dyadic observation histories that emerge from the presence and detection of

248 individuals, and the occurrence and detection of dyadic interactions between them. A capture-

249 recapture sampling design would in particular allow the explicit modeling of the link between the

250 frequency of contacts and the probably to miss the connection entirely. 


\section{Acknowledgements}

The data were procured from the online open source repository ASNR (Sah et al., 2019)

(https://github.com/bansallab/asnr). I am very grateful to the authors who made their data available on ASNR, to the creators of the repository, as well as Sebastian Sosa for suggesting it.

\section{Supplementary material}

Appendix S1: R script

\section{Literature cited}

Artime, O., Ramasco, J.J., San Miguel, M., 2017. Dynamics on networks: Competition of temporal and topological correlations. Sci. Rep. 7, 41627. https://doi.org/10.1038/srep41627

Balasubramaniam, K., Beisner, B., Guan, J., Vandeleest, J., Fushing, H., Atwill, E., McCowan, B., 2018. Social network community structure and the contact-mediated sharing of commensal E. coli among captive rhesus macaques (Macaca mulatta). PeerJ 6, e4271. https://doi.org/10.7717/peerj.4271

Barrat, A., Barthélemy, M., Pastor-Satorras, R., Vespignani, A., 2004. The architecture of complex weighted networks. Proc. Natl. Acad. Sci. U. S. A. 101, 3747-3752.

https://doi.org/10.1073/pnas.0400087101

Black, J.M., Owen, M., 1989. Agonistic behaviour in barnacle goose flocks: assessment, investment and reproductive success. Anim. Behav. 37, 199-209. https://doi.org/10.1016/00033472(89)90110-3

Butts, C.T., 2020. sna: Tools for Social Network Analysis. R package version 2.6.

Csardi, G., Nepusz, T., 2006. The igraph software package for complex network research. InterJournal, Complex Syst. 1695, 1-9. https://doi.org/10.3724/sp.j.1087.2009.02191

Franz, M., McLean, E., Tung, J., Altmann, J., Alberts, S.C., 2015. Self-organizing dominance hierarchies in a wild primate population. Proc. R. Soc. B Biol. Sci. 282, 20151512.

https://doi.org/10.1098/rspb.2015.1512

Granovetter, M.S., 1973. The Strength of Weak Ties. Am. J. Sociol. 78, 1360-1380.

Griffin, R.H., Nunn, C.L., 2012. Community structure and the spread of infectious disease in primate social networks. Evol. Ecol. 26, 779-800. https://doi.org/10.1007/s10682-011-9526-2

Kurvers, R.H.J.M., Adamczyk, V.M.A.P., Kraus, R.H.S., Hoffman, J.I., van Wieren, S.E., van der Jeugd, H.P., Amos, W., Prins, H.H.T., Jonker, R.M., 2013. Contrasting context dependence of familiarity and kinship in animal social networks. Anim. Behav. 86, 993-1001.

https://doi.org/10.1016/j.anbehav.2013.09.001

Lebreton, J.D., Nichols, J.D., Barker, R.J., Pradel, R., Spendelow, J.A., 2009. Modeling Individual Animal Histories with Multistate Capture-Recapture Models. Adv. Ecol. Res. 41, 87-173. https://doi.org/10.1016/S0065-2504(09)00403-6

Massen, J.J.M., Sterck, E.H.M., 2013. Stability and Durability of Intra- and Intersex Social Bonds of Captive Rhesus Macaques (Macaca mulatta). Int. J. Primatol. 34, 770-791. https://doi.org/10.1007/s10764-013-9695-7

Newman, M.E.J., Watts, D.J., Strogatz, S.H., 2002. Random graph models of social networks. Proc. Natl. Acad. Sci. U. S. A. 99, 2566-2572. 
Opsahl, T., Panzarasa, P., 2009. Clustering in weighted networks. Soc. Networks 31, 155-163. https://doi.org/10.1016/j.socnet.2009.02.002

Puga-Gonzalez, I., Ostner, J., Schülke, O., Sosa, S., Thierry, B., Sueur, C., 2018. Mechanisms of reciprocity and diversity in social networks: A modeling and comparative approach. Behav. Ecol. 29, 745-760. https://doi.org/10.1093/beheco/ary034

Sah, P., Méndez, J.D., Bansal, S., 2019. A multi-species repository of social networks. Sci. Data 6, 1-6. https://doi.org/10.1038/s41597-019-0056-z

Schank, T., Wagner, D., 2005. Finding, counting and listing all triangles in large graphs, an experimental study, in: Nikoletseas, S. (Ed.), Experimental and Efficient Algorithms. WEA 2005. Lecture Notes in Computer Science. Springer, Berlin, pp. 606-609. https://doi.org/10.1007/11427186_54

Silk, J.B., Beehner, J.C., Bergman, T.J., Crockford, C., Engh, A.L., Moscovice, L.R., Wittig, R.M., Seyfarth, R.M., Cheney, D.L., 2009. The benefits of social capital: close social bonds among female baboons enhance offspring survival. Proc. R. Soc. B Biol. Sci. 276, 3099-3104. https://doi.org/10.1098/rspb.2009.0681

Stahl, J., Tolsma, P.H., Loonen, M.J.J.E., Drent, R.H., 2001. Subordinates explore but dominants profit: resource competition in high Arctic barnacle goose flocks. Anim. Behav. 61, 257-264. https://doi.org/10.1006/anbe.2000.1564

Thierry, B., 2007. Unity in diversity: Lessons from macaque societies. Evol. Anthropol. 16, 224-238. https://doi.org/10.1002/evan.20147

Watts, D.J., Strogatz, S.H., 1998. Collective dynamics of "small-world" networks. Nature 393, 440442. https://doi. org/10.1038/30918 


\section{Figure legends}

Fig. 1: Triadic edge weight similarity and the four quadrants.
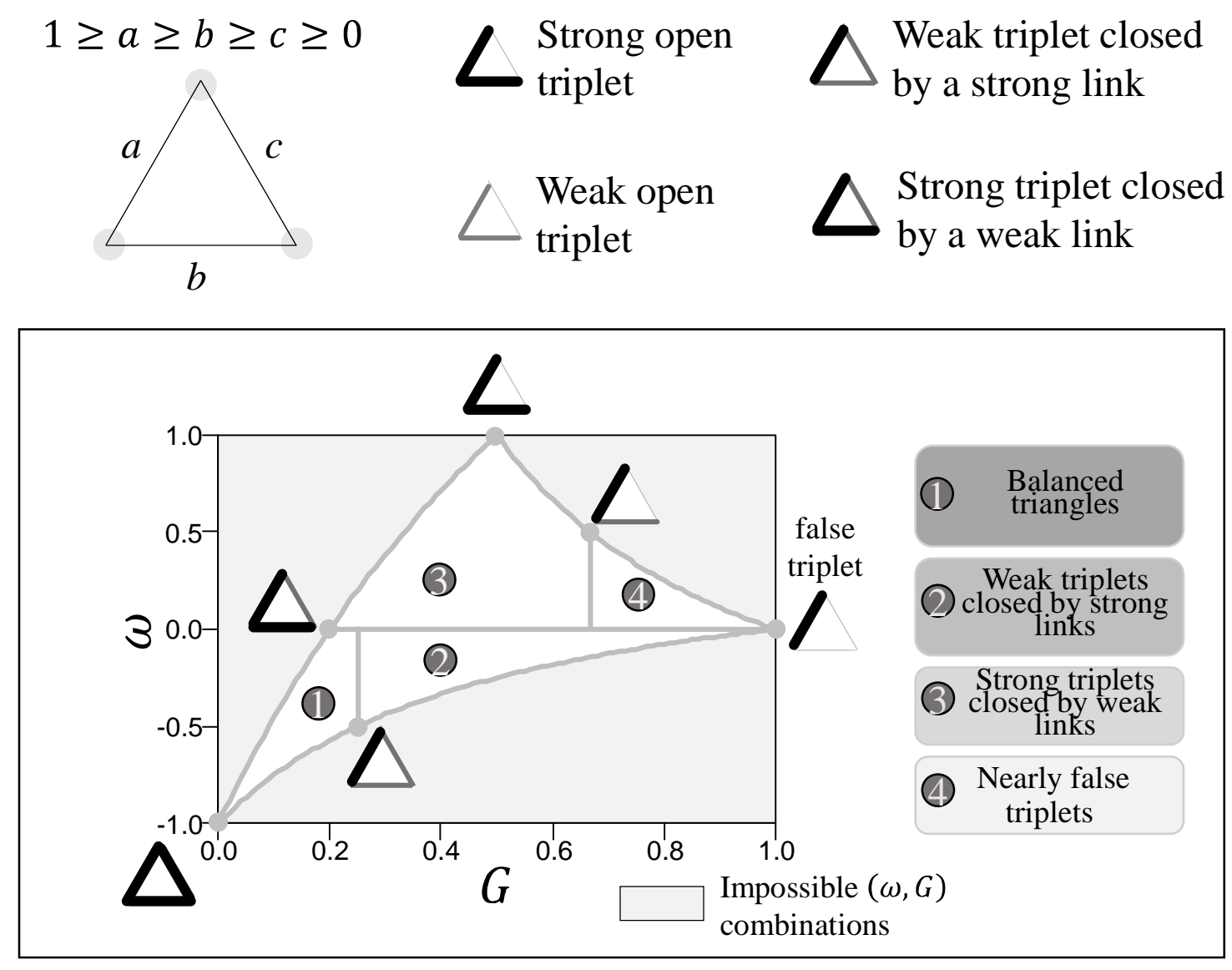
Fig. 2: Simulation results. (a) The usual transitivity scores, with notation as in the main text; the superscripts indicate the value of the threshold $c^{*}$ to decide when a link is significant. (b) The new transitivity scores. Scenarios 1-3 as explained in the main text. "full" = all the vertices are connected to each other but the strength of the connection varies according to a uniform or Gaussian law. "50\%" = same as "full" but half of the edges are removed. "Lattice": graph with a regular tiling pattern creating short-range neighborhoods for each vertex. I included lattices with 1 dimension (chain), 2 (square grid), 3 (cubic grid), and 4 dimensions. "Scale-free": networks whose degree distribution follows a power law, meaning that the vertex with the most connections has exponentially more connections than the vertex with the second most connections, and so on. "Modular + weak links": I created 2 to 8 full graphs with strong within-graph connections, then connected them to each other with edge weights below 0.1. "Star + weak link": all the vertices were connected to a central vertex with strong links, and then among themselves with edge weights below 0.1 .

(a) Usual transitivity scores, for different threshold values

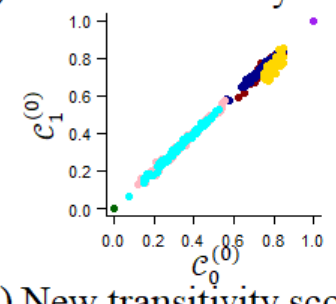

(b) New transitivity scores

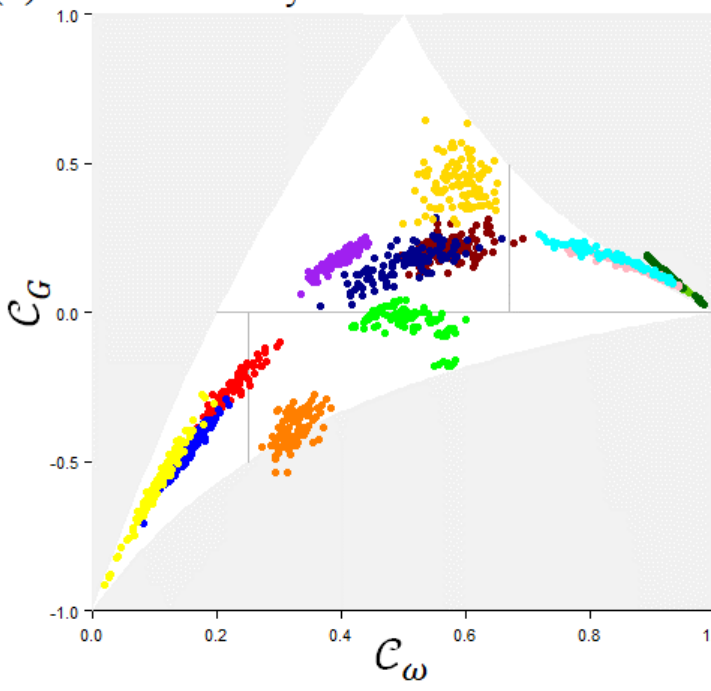

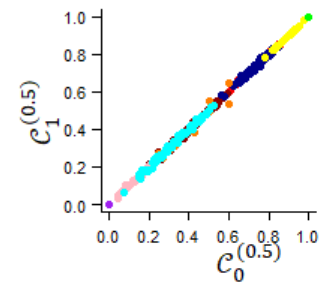

Scenario 1

- Scenario 2

- Scenario 3

- Full - uniform

- Full - Gaussian

- $50 \%$ - uniform

- $50 \%$ - Gaussian

- Lattice - uniform

- Lattice - Gaussian

- Scale-free - uniform

- Scale-free - Gaussian

- Modular + weak links

- Star + weak links 
Fig. 3: Case studies: graph-level scores. (a) The usual transitivity scores. (b) The new transitivity scores. The asterisks indicate significant distance tests.
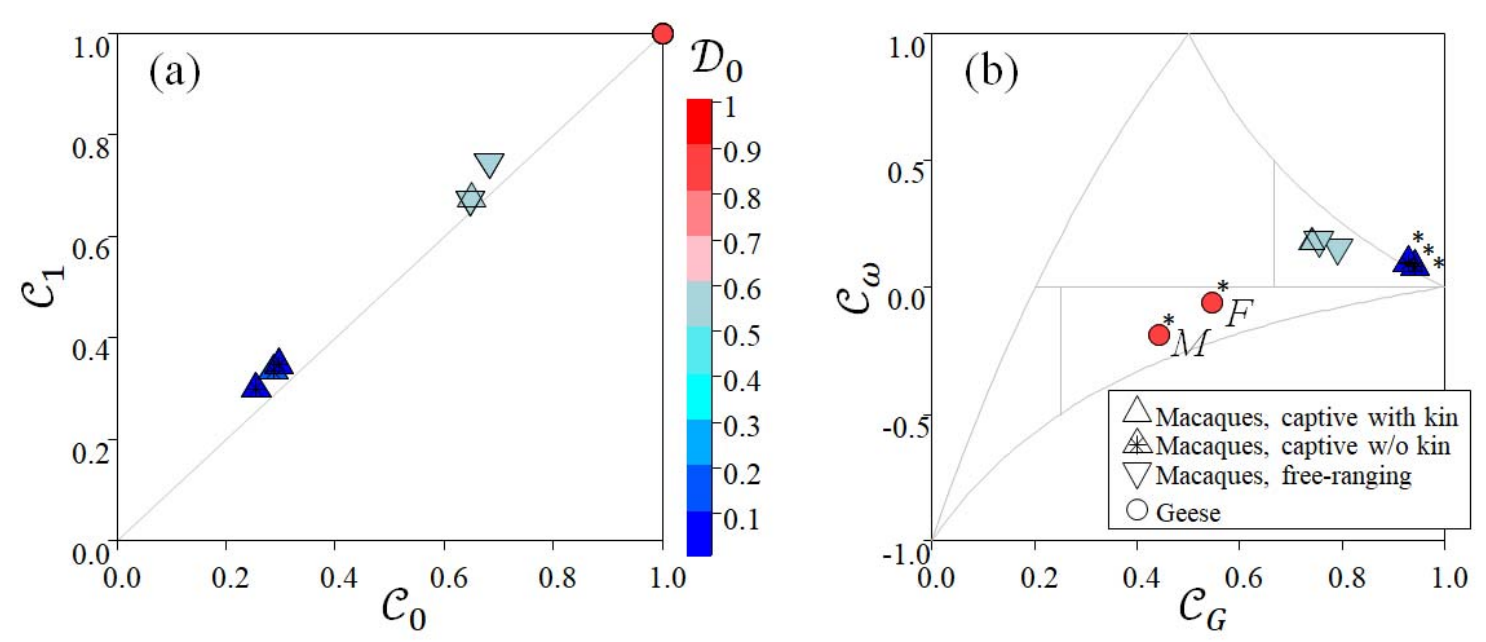

Fig. 4: Case studies: triplet-level scores. The triplets are divided into four quadrants as explained in Fig. 1. Grey histograms: observed distributions. Unfilled histograms: expected distributions at random, given the observed distributions of edge weights.

(a) Macaques, captive with kin

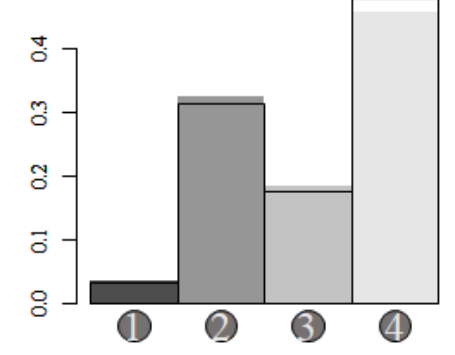

(c) Macaques, free-ranging

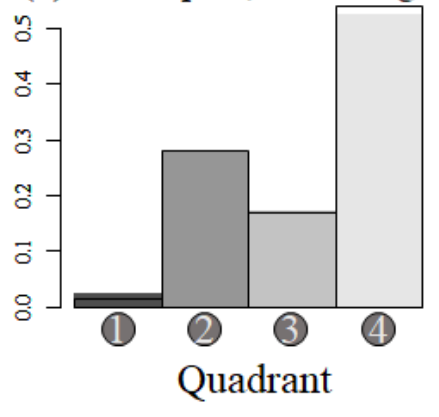

(b) Macaques, captive w/o kin

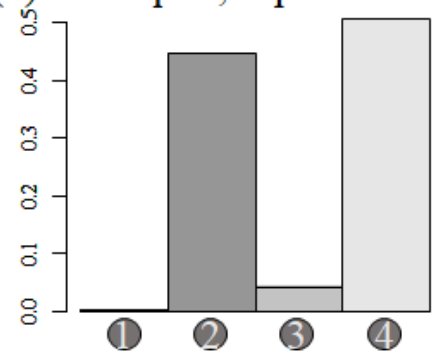

(d) Barnacle geese

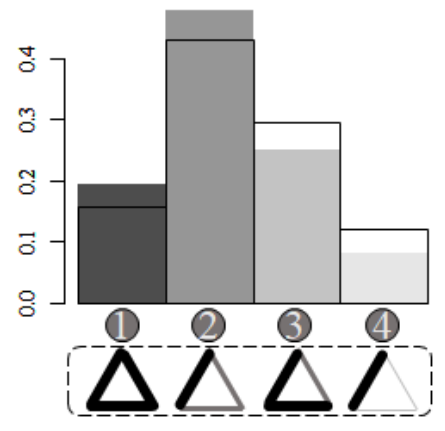

